\title{
XLII. Microscopic vision
}

\section{G. Johnstone Stoney M.A. D.Sc. F.R.S.}

To cite this article: G. Johnstone Stoney M.A. D.Sc. F.R.S. (1896) XLII. Microscopic vision , Philosophical Magazine Series 5, 42:258, 423-442, DOI: 10.1080/14786449608620934

To link to this article: http://dx.doi.org/10.1080/14786449608620934

$$
\text { 曲 Published online: } 08 \text { May } 2009 .
$$

Submit your article to this journal

Џ Article views: 2

Q View related articles ¿ 
above 1.8 and negative below this frequency, at 45 volts it was negative at all frequencies ; at 35 volts, however, it was positive at frequencies above $1 \cdot 8$, and also at frequencies below 1.8. That the resistance would be positive at all frequencies seemed probable, since the curves connecting the P.D. and current at constant arc-length for such carbons show that with small arc-lengths an increase of P.D. is accompanied by an increase of current.

The experiments have been carried out in the Physical Laboratory of the Central Technical College, and our best thanks are due to Professor Ayrton and $\mathrm{Mr}$. Mather for much kind advice during the course of the work.

\section{Note.-Fluctuation of the Current given by a Continuous- Current Dynamo.}

An attempt was made to replace the cells in Method I. by a dynamo, but it was found that even with the alternator at resi the electrostatic voltmeter showed a large deflexion. This was evidently due to the oscillation of the current owing to the commutator of the dynamo having a finite number of segments. The oscillation was found to increase as the brushes were moved out of the sparkless position. The dynamo was a 5 kilowatt 2-pole machine, and was giving 10 amperes at 70 volts. By adjusting the brushes to the sparkless position this oscillatory current, measured in the same way as in the case of the hissing are above, could not be reduced below 2.5 per cent. of the continuous current, while by rocking the brushes out of this position it could be increased to as much as 9 per cent. without the sparking at the brushes being excessive. The commutator was in good condition. This oscillation may introduce errors in the measurement by polarized instruments of the currents given by dynamos owing to the differences between the mean and the R.M.S. of such a current.

XLII. Microscopic Vision. By G. Johnstone Stoney, M.A., D.Sc., F.R.S.

[Continued from p. 349.]

\section{Addition to Parm I.}

[The following section should come into Part I. after Theorem 1, between $\$ \$ 7$ and 8.]

7 (a). Extension of Proposition 1.-In the proof of Proposition 1 it is assumed that the light emanates from each individual point of the object in an uninterrupted train of $2 \mathrm{H} 2$ 
hemispherical waves; whereas, in consequence of molecular events, the light really comes off in a broken succession of such trains, each of moderate duration and, generally, discontinuous from one train to the next. Within each train there must be such continuity as is manifested by the prolonged interference effects found in the use of such instruments as Rowland's largest diffraction gratings, or Michelson's Refractometer. But, to bring about these effects, the average length of the trains need not be more than some such length as a metric foot (30 centimetres) which includes about 500,000 luminous waves from the brightest part of the spectrum. This would correspond to an average duration of trains of about the thousand-millionth part of a second. But however brief the duration of each train, nevertheless for that short time the ætherial disturbance it occasions can be resolved in whatever ways it could have been resolved if the train had gone on for ever. Hence, in order to take into account the discontinuity of the trains of waves as actually emitted by the point $p$, it suffices simply to recognize that the undulations of uniform plane waves of Proposition 1 are made up of a succession of comparatively short sections of uniform plane waves, which may each include only some few thousand waves and which may be discontinuous where one succeeds another. This, however, does not hinder their continuing to be undulations of uniform plane waves: accordingly Proposition 1 extends to the cases where the emission of light from the points of the object is discontinuous.

[Corrections in Part I.-In the heading of $\$ 8, \mathrm{p} .338$, change "Principles of Reversal" into Principle of Reversal. In the footnote on p. 336 , line 7 , change "wave" into waves. In the diagram on p. 337 , the repetitions of the curve $m n$ should have been drawn of the same form as the original curve.]

\section{Part II.}

21. The Illuminating Apparatus.-The state in which light reaches that portion of a microscopic object which is under scrutiny is determined by the source of light, by the condenser, by the use that is made of the iris diaphragm and stops which are associated with the condenser, and by the parts of the microscopic object through which the light has to pass to reach the part which is being specially examined.

The iris diaphragm and stops determine the directions from which light is allowed to reach the object. These should be such as will bring into sufficient prominence in the image 
those features upon the object which we wish to examine, and at the same time such as will exclude or minimise the addition to the microscopic image of false or confusing effects. How this may best be aimed at will have to be considered in Part III., and will be illustrated there.

The source of light and the condenser are to be esteemed as good in the degree in which they enable the whole of the light of each wave-length which is brought to bear upon a point of the object to reach that point at each instant in the same phase and with accordant transversals. For if this condition could be fully attained the state in which the light would then leave the object would be complicated by the previous condition of the light only in respects which cancel one another when averaged over a sufficient period of time. For this average, 300 metres of cosmic time ${ }^{*}$ - the millionth of a secondwould be abundant. There is, therefore, no "twinkling of an eye" within which the average has not been struck. Hence light so supplied will furnish a pure image so far as man can see-one depending solely on the features of the object.

The efforts to reach this desirable result seem to have been directed exclusively towards improving the condenser, and cutting very thin sections; but both theory and experiment seem to show that when the condenser is good, a further advance may be made by attending to the source of light, which apparently ought to be confined to a layer of a thickness small compared with a wave-length, and preferably lying in a plane, or rather on a slightly concave surface, perpendicular to the optic axis (see $\$ 27$ below, p. 435). Of course the distanee of the source of light is not immaterial, since a well-corrected condenser acts at its best only when the source of light is at one particular distance.

However well the light may have been prepared by the condenser, it is sometimes thrown into confusion before it reaches the upper surface of the object, by having to enter through its under surface and to traverse its substance. In such eases the attempt to ascertain what is on the upper

* Cosmic time means time the portions of which are measured by lengths-by the distances over which electromagnetic waves in the open æther, and therefore orer which light in racuo, would travel in those portions of time. This way of measuring time is convenient in both optics and molecular physics. The convenience has its foundation in nature, as it no doubt arises out of the circumstance that this way of measuring time brings both time and space into the closest possible asscciation in which they can stand to the fundamental units of nature. See a paper "On the Physical Units of Nature" in the Proceedings of the Royal Dublin Society of Feb. 16, 1881, or in the Philosophical Magazine of May 1881 . 
surface is embarassed by somewhat similar difficulties to those we meet with when we try to look through ribbed glass. Thus, on the siliceous diatom known as Pinnularia nobilis, it is easy to make out that there are rows of dots on the tongues that stand inwards towards the median line; but vision is here mixed up with so many false effects, that it would be difficult to determine how many of these rows there are and where exactly they are placed. All will probably be reduced to order if the diatom can be mounted between two media, of which the under one shall have a refractive index of 1.4 , the same as silex, and the upper one a refractive index as much as possible exceeding 1.4. To mount objects in this way ought not to be impracticable.

22. The Six Images. - When a particular mode of illumination has been adopted, all subsequent events are thereby determined if the optical parts of the microscope are fully corrected and properly adjusted. To trace these events from the object on the stage to the image of it delineated upon the retina of the observer is now our task; and it may be prosecuted by beginning with an ideally perfect image and applying in succession a series of changes which finally transform it into the image which actually presents itself within the eye of the observer. Throughout the whole inquiry we may, without loss of generality, treat only of the light of one wavelength out of the light employed.

The following is a list of the successive stages of the transformation. In reading it the diagram on p. 433 will be found of use :-

A. Object $\mathrm{A}$ is the actual microscopic object, to which corresponds Image A, viz. : that ideal image reproducing all possible detail, which only light of infinitesimal wavelength supplied in a theoretically perfect manner would be eompetent to prodnce.

B. Image B, the standard image, means that image which the light of wave-length $\lambda$ as actually emitted by the microscopic object would furnish, if reversed (see Part I. section 8, p. 338). This image is of the same size as the microscopic object. To it would correspond Object $B$, an object of the same size as Object. A, but differing from it by containing no detail but that shown in Image $\mathrm{B}$.

C. Image C, standard image No. 2, is that which the light taken in by the objective would, if reversed, produce; to which would correspond Object $\mathrm{C}$, an object containing the same detail as that shown in Image C. 
Some of this detail may be in excess of any detail shown in Image B, but, unfortunately, an addition of this kind does not represent anything on the microscopic object (Part I., Proposition 3, p. 345).

Image $\mathrm{C}$ and Object $\mathrm{C}$, like Image $\mathrm{B}$ and Object $\mathrm{B}$, are of the same size as the microscopic object.

$\mathrm{D}$. Image $\mathrm{D}$, the focal image formed by the objective, which lies near the top of the tube of the microscope, is an enlargement with distortion of Image C. To it corresponds Object $D$, viz. that object which the focal image if seen would appear to be.

$\mathrm{E}$. Image $\mathrm{E}$, the risual image, is that virtual image which the eye-piece forms, when it is applied as a magnifying-glass to the focal image. It is an enlargement and slight distortion of the focal image. It is, therefore, an enlargement with a somewhat different distortion of lmage $\mathrm{C}$. To it corresponds Object $\mathrm{E}$, the visual object, that object which the observer thinks he sees.

$\mathrm{F}$. Finally Image $\mathrm{F}$ is the image actually produced on the retina of the observer.

The state in which the light has been admitted to the object affects images B, C, D, E and the corresponding objects, and therefore affects Image $\mathbf{F}$. We have now to advance in succession through these images, beginning with the ideal image and ending with the image which is actually formed within the eye of the observer.

23. Transition from $A$ to B.-Image A, an ideal image of the microscopical object, could only be formed by light of infinitesimal wave-length supplied in a theoretically perfect manner. This light, and this light alone, could form an image reproducing every detail. By such wave-lengths as can be used with the microscope-i.e. when we pass from Image A formed by imaginary light of infinitesimal wavelength to Image B formed out of real light_- the greater part of the events that happen in the object are shut out from our view by being massed together. In fact, when we come to a numerical example we shall find that a whole squadron of them, indeed an entire army, occupies that space upon the object which corresponds to the minutest speck which such light will show.

Image $\mathrm{B}$, the standard image, as appears from $\S 8$, p. 338, is formed by the flowing in upon it of beams of uniform plane waves; and in consequence it may, under Theorem 2, be regarded as formed of luminous rulings interlacing and interfering with one another : each luminous ruling being formed 
by the convergence of two or more of the above-mentioned beams of plane waves, or of components of them. The finest of these rulings are those formed by beams that flow in horizontally from opposite longitudes. If each of the beams is resolved into light polarized in, and light polarized perpendicularly to the plane of incidence, then the opposed pair of each of these components will produce a ruling in which the brightness varies by the law represented by the expression $1+\cos (4 \pi x / \lambda)$. This indicates that it is a ruling of which the spacing is $\lambda / 2$, each space including the width of a line and of the interval between it and the next. Such a ruling will be best seen when the intensity of the illumination is such that the lines and intervals appear of equal width. Hence the lines of the finest rulings, and consequently any speck in the image seen by the interlacing of these rulings, will have an apparent diameter of $\lambda / 4$, when best seen.

Now the corresponding portion of the microscopic object, viz. a globe with $\lambda / 4$ for its diameter, contains within it an enormous quantity of detail, all of which is massed together in the standard image and presented to us as one mere speck. How great a loss is here incurred, and how little any microscope can exhilitit to us of nature, may be judged from a numerical example which will be given in Part IIl.*

24. Transition from $B$ to $C$. - The important change from $B$ to $C$ requires to be carefully studied from many sides. Standard image No. 1 contains everything which the whole of the light of wave-length $\lambda$ is, in the condition in which it leaves the object, capable of presenting. Standard image No. 2 contains the utmost that can be delineated by that portion of this light which the objective is able to take in.

The change from the first of these to the second involves, in the first place, some obliteration of detail, consequent upon the exclusion of the inclined beams $\mathrm{B} a$, which are spoken of on p. 344, in $\$ 15$ of Part I. It also involves a rounding off

* The outcome of the computation here referred to is that with bluishgreen light, which has probably the shortest wave-length that can be used with advantage in eye observations, and when its wave-length is shortened by mounting the object in a medium with as high a refractive index as immersion oil : under these favcurable circumstances, a spherical portion of the object of the size of the smallest speck which this light is capable of exhibiting, would still be large enough to contain something between 300,000 and $300,000,000$ of those chemical atoms of which ponderable matter is made up. And we should remember that the immersion objective for which the oil is designed cannot exhibit quite so small a speck as the smallest which light of that wave-length could produce. It is a limit beyond its grasp. Biologists would do well to ponder what computations of this kind teach us. 
in the image of any sharp edges that may be upon the object, owing to the exclusion of the beams $\mathrm{B} b$. The exclusion of these same beams also often produces a false glare, or adds features to the image that are foreign to the object (Proposition 3, p. 345). We shall have to study of what kinds these illusory effects are, and how they may be in some degreo controlled by adjustments of the illuminating apparatus. There is another consequence of the exclusion of the beams $\mathbf{B} a$ and $\mathbf{B} b$. It is apt to produce a deceptive colouration of the images seen in the field of view. (Proposition 4,p. 345.)

The most important part of the study of microscopic vision is the study of these matters. But before endeavouring to go more fully into them, which can be best done in Part III., we must take a glance at the subsequent and less important steps that intervene between Standard Image No. 2 and the Visual Image, which last is what is directly presented to the eye of the observer.

25. Transition from $C$ to $D$. - It has been proved in the footnote on p. 339 of Part I., that the illuminating apparatus and the microscopic object may be removed, and that Standard Image No. 1 can take their place. Now, for our present purpose it will be enough to put Standard Image No. 2 in their place ; since we are only concerned with the light which is to be made use of by the objective, and this light remains the same whether it is supplied by standard image No. 1 or standard image No. 2. In order to be available for this use of it, standard image No. 2 must be regarded as formed by subjecting that portion of the light emitted by the object which can be taken in by the objective to two successive reversals; and we shall find it convenient to conceive the medium in front of the objective, whether oil or air, to be continued downwards in order that the image may lie within this medium, so that the light may be able to go straight from the image to the objective. After the second reversal the light first forms the image and then proceeds on from it and enters the objective in precisely the same state as the light actually emitted by the microscopic object would have done. We may therefore remove the source of light and the microscopic object, and substitute for them standard image No. 2, transmitting the beams that form it on to the objective. Each of these beams is a beam of parallel light, and is therefore, by passing through the objective, brought to a focus at $x$, a situation which, is usually close to the back lens of the objective. See the figure on p. 433. After passing this focus the beam diverges in the form of a cone of convex spherical waves, and it is in this state that it reaches $D$, the 
place where the objective forms its image of the microscopic object.

This is what happens to one of the beams. Let us still farther confine our attention to the course pursued by its axial ray, i. e. the ray which coincides with the line drawn from the middle of the objective field perpendicular to those plane waves which form the beam before entering the objective. This axial ray starts from the point on the optic axis of the microscope where this optic axis pierces image $\mathrm{C}$. When the course of the ray is traced through and past the objective it is found a second time to intersect the optic axis, at the point where this axis pierces image $\mathrm{D}$, the focal image. Let $\alpha$ and $\beta$ be its inclinations to the optic axis before and after it passes through the objective. Then by Lagrange's Theorem

$$
n \sin \alpha=\mathrm{M} \sin \beta, \text {. . . . . . . }
$$

where $\mathrm{M}$ is the magnifying power of the objective, i.e. the number of times that the image $\mathrm{D}$ is larger than the image $\mathrm{C}$; and where $n$ is the refractive index of the medium between $\mathrm{C}$ and the objective, the refractive index of the air which intervenes between the objective and D being taken as unity [otherwise $n$ would have to be the ratio of the two refractive indices].

Now if we take two beams, whose axial rays are in the same meridian plane*, and which are inclined to the optic axis at angles $\alpha$ and $\alpha^{\prime}$, then the ruling in image $\mathrm{C}$, to which they will give rise, has a spacing

$$
\epsilon_{1}=\lambda_{1} /\left(\sin \alpha-\sin \alpha^{\prime}\right), \text {. . . . . . }
$$

$\lambda_{1}$ being the wave-length in the medium which is in front of the objective. Similarly the spacing of the ruling which these same beams produce when they reach image $D$ is

$$
\epsilon_{2}=\lambda_{2} /\left(\sin \beta-\sin \beta^{\prime}\right), \text {. . . . . . }
$$

where $\lambda_{2}$ is the wave-length in air. Hence we find that the ratio of $\epsilon_{2}$ to $\epsilon_{1}$ is

$$
\frac{\epsilon_{2}}{\epsilon_{1}}=\frac{\lambda_{2}\left(\sin \alpha-\sin \alpha^{\prime}\right)}{\lambda_{1}\left(\sin \beta-\sin \beta^{\prime}\right)} . \text {. . . . . }
$$

and this, when we replace $\lambda_{2} / \lambda_{1}$ by $n$, and put in the values of $n \sin \alpha$ and $n \sin \alpha^{\prime}$ given by equation (1), becomes

$$
\frac{\epsilon_{2}}{\epsilon_{1}}=\mathrm{M}, \quad \text {. . . . . . . . . . . }
$$

* A meridian plane means a plane passing through the optic axis of the microscope. 
The process that has been here followed might in fact have been employed to prove Lagrange's Theorem ; but it is here used for the purpose of showing that the ratio $\epsilon_{2} / \epsilon_{1}$ is independent of the values of $\alpha$ and $\alpha^{\prime}$ : in other words, that all the rulings of which image $\mathrm{C}$ is formed are reproduced on a larger scale in image $\mathrm{D}$, and that the scale of the enlargement is the same for them all.

We thus learn that image $\mathrm{D}$ is formed of exactly the same rulings as image $\mathrm{C}$, only magnified and somewhat distorted-. distorted laterally by the convex form of the beams, and longitudinally owing to the decreased inclination of the beams to one another. Inage $D$ accordingly contains every feature which is present in image $C$, only somewhat distorted laterally, and still more distorted longitudinally.

Hence the great task we have to set before ourselves is to find out what image C, standard image No. 2, contains. This conclusion is not disturbed by pursuing the course of events farther.

26. From $D$ to $E$, and from $E$ to $F$.-The subsequent stages need not detain us long. If, as before, we select two of the beams of plane waves emitted by standard image No. 2, and if we follow the course of the axial rays of these two beams, we find that these rays intersect the optic axis where this axis pierces images $\mathrm{C}, \mathrm{D}$, and $\mathrm{F}$ (see the figure on p. 433), and that if the portions of them which lie between the eyepiece and the eye were produced backwards they would also intersect the optic axis where that axis pierces the image $E$, which is a virtual image. This last is the Visual Image, $i . e$. the image which seems to the observer to be presented to him.

Let $\alpha \alpha^{\prime}, \beta \beta^{\prime}, \gamma \gamma^{\prime}$, and $\delta \delta^{\prime}$ be the angles at which the axial rays of the two beams intersect the axis at $\mathrm{C}, \mathrm{D}, \mathrm{E}$, and $\mathrm{F}$. At each of these images the two beams give rise to a ruling, and if the spacings of these rulings in the successive images be designated by $\epsilon_{1}, \epsilon_{2}, \epsilon_{3}, \epsilon_{4}$, we have, by proceeding as in $\S 25$,

$$
\epsilon_{2}=M \epsilon_{1}, \quad \epsilon_{3}=M^{\prime} \epsilon_{1}, \quad \epsilon_{4}=M^{\prime \prime} \epsilon_{1} \quad \text {. . }
$$

where $M, M^{\prime}$, and $M^{\prime \prime}$ are the number of times that images $D$, $\mathrm{E}$, and $\mathrm{F}$ respectively are larger than the mieroscopic object; and where also, by equation (1),

$$
\left.\begin{array}{l}
\mathbf{M}=n \sin \alpha / \sin \beta, \text { and is also }=n \sin \alpha^{\prime} / \sin \beta^{\prime} \\
\mathbf{M}^{\prime}=n \sin \alpha / \sin \gamma, \text { and is also }=n \sin \alpha^{\prime} / \sin \gamma^{\prime} \\
\mathbf{M}^{\prime \prime}=n \sin \alpha / n^{\prime} \sin \delta, \text { and is also }=n \sin \alpha^{\prime} / n^{\prime} \sin \delta^{\prime}
\end{array}\right\} .
$$


where $n^{*}, 1$, and $n^{\prime}$ are respectively the indices of refraction of the medium in front of the objective, of air, and of the vitreous humour in the eye of the observer.

Hence the ruling to which these two beams give rise in image $\mathrm{C}$ is reproduced in images $\mathrm{D}, \mathrm{E}$, and $\mathrm{F}$. The same is true of all the rulings by the co-existence of which image $\mathrm{C}$ is formed; and as within each image the spacings of all the rulings are modified in the same way, it follows that every feature which is present in any one of these images is present in all the others- of course on the supposition that no defect is introduced by imperfections in the objective, the eyepiece, or the eye.

Image $\mathrm{E}$ appears to the observer like an object presented to his unassisted eye, and in it the conditions must be fulfilled for naked-eye vision. These chiefly concern the angular size which that image must have in order that the minutest detail in it may be sufficiently large to be satisfactorily seen (see Section I. of a paper "On the Limits of Vision," Scientific Proceedings of the Royal Dublin Society of December 20, 1893 , p. 228 ; or, Philosophical Magazine for March 1894, p. 317).

But as to what the detail is, and as to the true nature of that detail, we still have to fall back upon a study of standard image No. 2, and of the adjustments of the microscope which are available for improving that image. And this can be best done by the study of individual examples, which is to be the subject matter of Part III.

If any difficulty still hangs over the subject treated of in the last few sections it will be cleared up by following out the whole history of one individual beam.

27. Course of an individual beam traced, and How to see $i t$. - It has already been proved that the microscopic object and all the illuminating apparatus may be removed, and that standard image No. 2 may be substituted for them, if this image be formed after two reversals, and if it therefore emits its light forwards. Let $\mathrm{C}$ in the figure be the position of this staudard image and let $\mathrm{C} b$ be one of the beams of uniform plane waves which it sends forward. Let the dark line in the figure represent the axial ray, that is the ray which starting from the middle of image $\mathrm{C}$ continues throughout perpendicular to the waves of which the beam consists. The

* It is the usual practice in England to employ the symbol $\mu$ for the refractive index: but it cannot conveniently be so used in treating of the microscope, since $\mu$ is wanted as the designation of the micron. On this account the continental practice of using $n$ for the refractive index has been adopted in the text. 
beam enters the objective at $b$, and by the objective is brought to a focus at $x$, the focus for parallel rays. Here it forms an Airy's spurious disk, or rather a somewhat more diffuse spurious disk, since the objective falls short of being aplanatic in its treatment of beams of parallel light incident on its front surface. Past the focus $x$ the beam advances, and diverges as it advances. Let $\mathrm{D}$ be where the axial ray, the dark line of the figure, intersects the optic axis of the microscope. Note that the axial rays of all the other beams enitted from the image $\mathrm{C}$ will, like this one, start from the point where $\mathrm{C}$ is pierced by the optic axis of the microscope, and will all intersect that axis again at $D$. At $D$ an image of $\mathrm{C}$ is formed on a somewhat curved surface that stands at right angles to the optic axis of the microscope. Our beam, diverging from $x$, when it reaches this surface contributes its quota towards the formation of the image upon it. After passing image $\mathrm{D}$ the divergent beam continues till it reaches the front of the eyepiece. It proceeds through

Fig. 1.

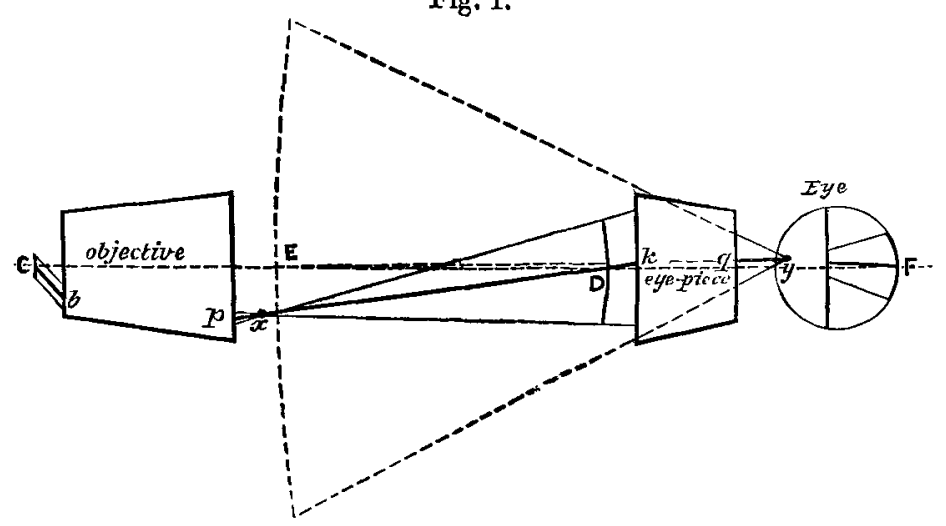

the eyepiece and is by it brought to a second focus at $y$, where the eyepiece can form an image of $x$. If we produce the portion $q y$ of the axial ray backwards, it will intersect the optic axis at $\mathbf{E}$, where the backward production of the convergent beam $q y$ will widen out so as to extend over the space occupied by the virtual image $\mathrm{E}$, and contribute its share towards the formation of that image. Accordingly the light of our beam, which advances in the direction $q y$, will enter the eye (the pupil of which should be brought close to $y$ ) in the same state as if it had come direct from the whole extent of an image at E. After passing $y$ the beam again diverges, and by the optical action of the front half of 
the eye this divergence is lessened and at the same time the axial ray is bent in so as a third time to intersect the optic axis of the microscope, where that axis prolonged pierces the retina of the observer. Upon the retina, round this point, the beam of lessened divergence spreads and there assists in the formation of image $\mathrm{F}$, the image of the microscopic object which is formed by the microscope upon the retina of the observer.

Twice upon its journey the beam we are dealing with has been brought to a focus-at $x$ and at $y$. At both these positions it is concentrated into a point or, to use more accurate language, into a spurious disk, and can be seen as such. It may be seen at $x$ by removing the eyepiece and looking down the tube of the microscope, taking care to keep the eye central ; and it may also be seen at $y$ by replacing the eyepiece and looking with a magnifier at the image $y$ formed in the air outside the eyepiece *.

Accordingly every visible point of the image seen at $x$ when we look down the tube of the microscope is the concentrated light of one of the beams of uniform plane waves which has been emitted from the whole extent of the objective field. We may therefore, by scrutinizing the position and brightness of the points of this image, learn in what directions and with what intensity the beams of uniform plane waves are being thrown off from the whole front of the objective field.

$\mathrm{W}^{T}$ e must not, however, suppose that by scrutinizing this image we can learn everything about those beams, inasmuch as they differ from one another not only in those respects of which the eye can take cognizance, but also in the form and position of the elliptic motion in each wave front, and in its phase at a given instant-particulars which, though the eye cannot detect them, are of primary importance in determining what image of the microscopic object they will contribute to form when they reach the position $D$.

To complete this survey, we should trace the course of the light up to the microscopic object, as well as from that position forward. The light as it reaches the objective field would be ideally perfect if it could be resolved into beams of plane waves, perfectly uniform, coming with equal intensity

* The effect is most striking when the object is such as directs into a few definite beams each beam of the light supplied to it by the condenser. An excellent objectis the diatom known as Peristephania eutycha, especially when the iris diaphragm is nearly closed, so that only a small sheaf of beams, each extending over the whole objective field, is supplied by the condenser. 
Irom all the directions in which the iris diaphragm and stops allow light to pass, and at the same time in all other respects -in phase, ellipticity, and so on-such light as would have been emitted in the reverse direction downwards from $a$ perfectly featureless self-luminous plane occupying the position upon the stage of the microscope of the objective field.

This ideal is the more nearly approximated to, the better the condenser; and it would appear that the best position of the source of light is that which would be occupied by the image which the condenser would form beneath of such a featureless luminous plane as we have supposed. Hence the suggestion as to the position of the source of light made in $\$ 21$. It further appears that the ideal position for the iris diaphragm and stops would be at the position which we may call $z$ (corresponding to $x$ and $y$ ) where beams of uniform plane waves emitted downwards from the supposed luminous plane would be brought to a focus by the condenser. This is a position which is usually very close to the condenser; and it would be a marked improvement in microscopes if the iris diaphragm and stops were brought nearer to this ideal position than they commonly are. As they are at present placed, different parts of the field of view are treated differently by them in an appreciable degree.

We have next to examine into some points the consideration of which will put us in a better position for interpreting aright what we shall see in the microscope.

28. Of the Composition and Resolution of Undulations.-We shall start from the known fact that any luminous undulation of uniform plane waves of wave-length $\lambda$ may be resolved into two undulations of plane-polarized light polarized in planes at right angles. In order to get their equations in their simplest form, let the axis of $x$ be placed perpendicular to the wave surfaces, and the axes of $y$ and $z$ parallel to the transversals of the two plane-polarized components. Then the equations of the given undulation, which we may call $U$, will be-

$$
\begin{aligned}
& \eta=a \sin \left(\frac{2 \pi}{\lambda}(v t-x)+\alpha\right), . . . \\
& \zeta=b \sin \left(\frac{2 \pi}{\lambda}(v t-x)+\beta\right), . .
\end{aligned}
$$

in which $\eta$ and $\zeta$ are the displacements, at the time $t$, in the two plane-polarized components, $a$ and $b$ are the amplitudes (i.e. the scalar part of the transversals), $v$ is the velocity of light, and $\alpha$ and $\beta$ are the initial phxses on the plane $y z$, 
which last are constants that are determined by the ellipticity of the light of the undulation $U$, by the position of its ellipse, and by its initial phase.

Similarly, another undulation $\mathrm{V}$ of uniform plane waves travelling in the same direction will be represented by equations 3 and 4 -

$$
\begin{aligned}
\eta^{\prime} & =a^{\prime} \sin \left(\frac{2 \pi}{\lambda}(v t-x)+\alpha^{\prime}\right), \ldots . . \\
\xi^{\prime} & =b^{\prime} \sin \left(\frac{2 \pi}{\lambda}(v t-x)+\beta^{\prime}\right) \ldots . .
\end{aligned}
$$

If the undulations $U$ and $V$ are simultaneously present, the displacements over the plane $x$ and at the time $t$ will be $\eta+\eta^{\prime}$ and $\zeta+\zeta^{\prime}$; and these by elementary trigonometry are found to be

where

$$
\begin{aligned}
\eta+\eta^{\prime} & =\mathrm{M} \sin \left(\frac{2 \pi}{\lambda}(v t-x)+\mathrm{A}\right), \ldots . \\
\zeta+\zeta^{\prime} & =\mathrm{N} \sin \left(\frac{2 \pi}{\lambda}(v t-x)+\mathrm{B}\right), \ldots .
\end{aligned}
$$

$$
\begin{gathered}
\mathrm{M}^{2}=a^{2}+a^{\prime 2}+2 a a^{\prime} \cos \left(\alpha-\alpha^{\prime}\right), \ldots \\
\tan \mathrm{A}=\frac{a \sin \alpha+a^{\prime} \sin \alpha^{\prime}}{a \cos \alpha+a^{\prime} \cos \alpha^{\prime}}, \ldots . \\
\mathrm{N}^{2}=b^{2}+b^{\prime 2}+2 b b^{\prime} \cos \left(\beta-\beta^{\prime}\right), \\
\tan \mathrm{B}=\frac{b \sin \beta+b^{\prime} \sin \beta^{\prime}}{b \cos \beta+b^{\prime} \cos \beta^{\prime}} .
\end{gathered}
$$

Equations (5) and (6) represent an undulation of uniform plane waves travelling in the same direction as $\mathrm{U}$ and $\mathrm{V}$. We may call this resultant undulation W. Equations (7), $(8),(9),(10)$ enable us to determine the constants of any one of these three undulations, if we know those of the other two. It appears accordingly that any two of the three undulations $\mathrm{U}, \mathrm{V}, \mathrm{W}$ being given, the third can be found.

It is an easy inference from this that any number of undulations of aniform plane waves of wave-length $\lambda$, that travel in the same direction, may be combined into a single undulation of the same kind travelling in that direction : a proposition of which use was made above in the latter part of $\S 6$, of Part I., p. 337.

29. Of elementary sheafs of beams, and of the single beams which may be substituted for them.-Beams of uniform plane waves may be emitted in any or all directions from the front 
of the objective field, or from either of its "standard images." Draw wbat we have called the axial rays of these. They are lines radiating from the middle of the objective field, and each perpendicular to the waves of its own beam. Take a group of these axial rays which lie within a definite cone, then the corresponding beams may be called a sheaf of beams; and where the cone within which the axial rays are confined is a very acute one, the corresponding beams may be called an elementary sheaf of beams. The whole of the beams emitted by the objective field, or from either of its standard images, may obviously be conceived of as divided up into e!ementary sheafs of any required degree of minuteness.

If we only have to deal with an image of limited extent like standard image No. 1, or standard image No. 2 (which are the same size as the objective field), then we are justified in substituting a single beam travelling along the axis of the cone for each elementary sheaf of beams. This may be proved as follows :-

Let $U$ be one of the beams whose axial ray lies within the elementary cone, and let $\theta$ be the angle between that axial ray and the axis of the cone. The cone, of course, has its vertex at the centre of the objective field. Let now $\mathrm{V}$ be an equivalent beam whose axial ray lies along the axis of the cone, and let the phase of $\mathrm{V}$ be such that $\mathrm{U}$ and $\mathrm{V}$ are in the same phase at the centre of the objective field. Then, as in $\S 15$, let $-V$ mean the same beam as $+V$, only with $\pi$ added to all its phases. Accordingly, if $+V$ and $-V$ are simultaneously present they cancel one another absolutely. We may therefore add hoth of these to the elementary sheaf of beams without altering it. Now $-\mathrm{V}$ and $\mathrm{U}$ would produce a ruling which will be the coarser, i.e. with its luminous bands more widely spaced, the smaller the angle $\theta$ is. Moreover, since $+V$ and $U$ are in the same phase at the vertex of the cone, which is also the centre of the objective field, it follows that one of the minima of illumination of the ruling produced by $-\mathrm{V}$ and $\mathrm{U}$ will occupy that position. Now by making $\theta$ sufficiently small, the spacing of this ruling may be made so many times larger than the objective field that there is no appreciable illumination anywhere within the limits of the objective field. If this be so, we may suppress the beams $-\mathrm{V}$ and $\mathrm{U}$ without producing appreciable change within the limits of the objective field. When this is done, the elementary sheaf of beams differs from what it was at first by baving $+\forall$ now in the place of $U$. By a similar process we may substitute $V^{\prime}, V^{\prime \prime}$, \&c. travelling along the axis of the elementary cone for the other beams whose axial rays lie within the Phil. Mag. S. 5. Vol. 42. No. 258. Nov. 1896. 2 I 
cone. And, finally, all these V's, since they travel in precisely the same direction, will, as is proved in the last section, coalesce into a single resultant beam $\mathrm{W}$ travelling along the axis of the cone, which single resultant may accordingly be substituted for the elementary sheaf of beams.

The general conclusion is :- The whole of the light emitted from the objective field may, by Theorem 1, bo resolved into beams of uniform plane waves; these beams may be divided into small groups, each an elementary sheaf of beams; and each elementary sheaf of beams may have a single beam substituted for it.- In every subsequent step of our investigation we need only deal with these resultants-these secondary beains as they may be called-which, though many, are limited in number.

30. Another proof of Theorem 2.- Theorem 2 may be proved in many ways, and a proof which carries the analysis of an image down to its simplest elements will be found instructive. Describe a hemisphere in front of the objective field and round its centre. Call the point where the optic Fig. 2.

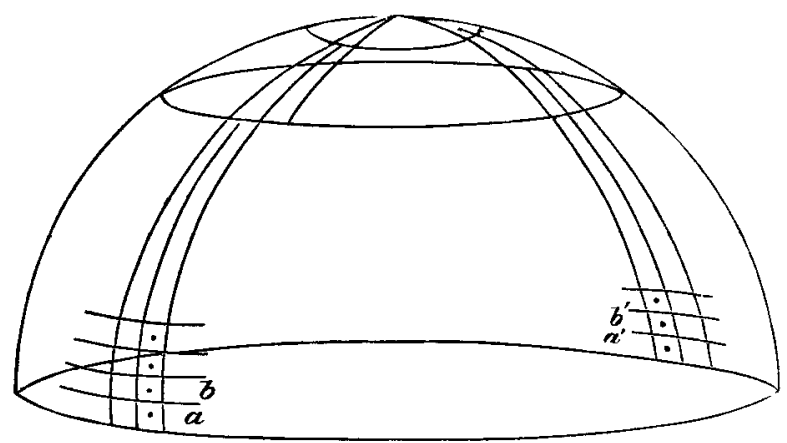

axis of the microscope pierces this hemisphere, its pole. Planes passing through the optic axis may be called the meridional planes; and the objective plane, being perpendicular to the axis of the microscope, will be its equatorial plane. Divide the equator of our hemisphere into seconds of arc, i. e., into 1,296,000 parts, which will afford sufficiently minute divisions upon which the bases of elementary cones may abut. Draw parallels of latitude also at intervals of a second ; and draw meridians as in the figure, marking out in conjunction with the parallels of latitude the bases of the elementary cones, or rather pyramids. These become narrower the bigher the latitude, and as soon as they bave shrunk to half a second horizontally every alternate meridian may be omitted, until they have shrunk again till other meridians may be omitted without any of the little sectors 
being more than a second in width. By this process the whole hemisphere may be mapped out into these patches placed in vertical series, each series with a corresponding series on the opposite side of the polar axis, and the whole ending up in one patch or in one pair of patches at the pole. From the middle of each patch draw a radius of the sphere, omitted from the diagram to avoid confusion, but easily conceived. These are the axial rays of the secondary beams $W$, which we are at liberty to substitute for the elementary sheafs of beams that really exist.

In this as in all similar problems it is convenient to begin by resolving each beam of light into two plane-polarized beams, one polarized in and the other perpendicular to the meridional plane in which its axial ray lies. We may then confine our attention first to the light polarized in one of these ways. Let us then call the secondary beams which are polarized in one of these ways $a, b, \&$ c., and the corresponding beams opposite to them and polarized in the same way, $a^{\prime}, b^{\prime}$, \&c., as in the figure.

In general, beam $a$ and beam $a^{\prime}$ will not be alike. However, heam $a^{\prime}$ may by the proposition in $\$ 23$, p. 435 , be subdivided into $a_{1}^{\prime}$ and $a_{2}^{\prime}$, of which $a_{1}^{\prime}$ shall be exactly similar to $a$. Accordingly $a$ and $a_{1}^{\prime}$ produce a definite ruling of equal and equidistant lines extending over the whole standard image ; and $a_{2}^{\prime}$ has still to be disposed of. Resolve $b$ into two beams of which $b_{1}$ is similar to $a_{2}^{\prime}$, and $b_{2}$ is the other component. Then $a_{2}^{\prime}$ and $b_{1}$ produce another ruling, and $b_{2}$ has next to be disposed of. 'l'o do this, resolve $b^{\prime}$ into $b^{\prime}$, and $b_{2}^{\prime}$, of which $b_{1}^{\prime}$ is similar to $b_{2}$. Then $b_{2}$ and $b_{1}^{\prime}$ produce a ruling and $b_{2}^{\prime}$ is what remains over. By continuing this process the whole of the vertical columns over $a$ and $a^{\prime}$ may be dispose:l of : and in a similar way the other columns can be treated. All the rulings extend over the whole of the standard image, and between them use up the whole of the light polarized one way, except that residium which is left over in the last patch at the pole. This residium can be made as small as we please by diminishing the size of our elementary cones; and of whatever amount it is, it only represents the limiting case of a ruling produced by two beams advancing at a vavishing angle to one another, and of which accordingly the spacing is infinite.

A similar treatment applies to the light polarized the other way, which also produces its body of rulings; so that finally the whole of the light emitted by the objective field, however varied its contents, can be laid down on the standard image in the form of a number of luminous rulings of uniform parallel lines, each of them of that optically most simple character 212 
which is produced by a single pair of beams that are alike and polarized, eitlier both in the plane of incidence or both perpendicularly to it.

A similar treatment applies to standard image No. 2, the only difference being that to analyse it we are to employ a sector of the hemisphere in the figure on p. 438 instead of the whole hemisphere.

Hence Theorem 2 is fully proved.

31. The resolution not unique.-In order to follow the most simple process when combining the secondary beams in pairs, we have taken both the members of each pair from the same meridian. We might of course have combined them laterally or obliquely, and we shall find it necessary to bear this in mind when dealing with some kinds of illumination that are found useful, e.g. annular illumination. It is obvious that it is legitimate to combine the secondary beams in any way which when completed has used up all the light : and our object should be to combine them in each case in whatever order is most convenient for the problem in hand. In the practical use of the microscope it is usually quite easy to see into what groups it is most advantageous to throw them. In whatever order they are taken the final result is the same; but one order differs from another in the degree in which it gives us information that is of use to us.

It is sometimes convenient to think of an optical image as a kind of picture, and that the rulings are, as it were, successively painted in upon the field of view to form it. But if we conceive matters in this way we must remember that this luminous paint behaves after a very peculiar fashion. Where one ruling crosses another or overlies it, they may obliterate one another in some parts as well as strengthen one another in others, effects which will depend on the lengths and positions of the transversals in the two rulings and upon the relation in which their phases stand to one another.

In this connexion it is very necessary to bear in mind that two rulings may be seemingly identical-i. e., identical in position, spacing, intensity, \&c., in such matters as the eye can perceive-and yet these rulings may behave quite differently towards the other rulings with which they are associated, owing to differences affecting the transversals and phases which our eyes are not fitted to take note of. Thus, what are apparently identical rulings might result from the interference of two beams little inclined to the optic axis, and from two others much inclined; or from two beans in the same meridian and two others in other positions : but these seemingly identical rulings would all behave differently towards the rest of the light with which they have to act. 
32. Criticism of the Albe and the Airy methods.-The proof which we have just gone through is instructive in many ways. In the first place, it carries the analysis of the image down to rulings of the simplest kind that are known. In the second place, it makes the flexibility and Protean character of the whole process conspicuous ; for although we followed one particular order in combining the secondary beams in pairs to form rulings, it is manifest that this order was in no degree necessary, and that the secondary beams might have been grouped in an infinite number: of different ways, the only condition being that whatever order we adopt we must take care to use up all the light. This means that the set of rulings which form an image is not unique : that there are an infinite number of such sets, any one of which will suffice to form the image.

But, above all, the proof given in the last section brings well into view the source of the advantage which is found in employing Abbe's mode of procedure as our instrument for searehing out the causes of the phenomena presented by microscopic vision. By the process we have followed the light sent forward from the objective field has been analysed into beams of plane waves. Now this is only one of numberless possible ways of analysing that light. It is an analysis which may be made, not which must be made. But it has the advantage over every other analysis, that the resulting waves are uniform waves, exactly alike in every respect over the whole extent of each wave surface and each wave retaining its form and intensity unchanged during its advance.

This sets us free from a sea of difficulties that embarass our progress when we attempt to employ any other resolution. If the analysis be made into curved waves of any kind-into those of Airy's method or any others-the resulting waves are not uniform over each wave-surface; and as the law of this want of uniformity is not yet known, we can only legitimately employ Airy's method in the cases where this want of uniformity has an inconspicuous effect upon the result of our inquiry. This is the case, for instance, in the treatment of telescopic vision to which Airy applied it. Here the sector of each hemispherical wave that we have to deal with is sufficiently small for the want of uniformity within its small extent to be of negligible amount.

It has sometimes been supposed that we can investigate microscopic vision by applying Airy's analysis to the light sent forward to the eye from the image of the microscopical object which is formed by the objective close to the eyepiece. This light as it comes from each point of that image is confined within a cone which is a continuation upwards of the nazrow 
cone from the back lens of the objective to that point of the image. Accordingly, if that object which is called the visual substitute in Part I., $\S 14$, p. 342, could be put in place of this image, sending forward hemispherical waves from each point of it which might be treated as uniform within the limits of the aforesaid cone; then what the Airy method as hitherto applied has investigated is what minuteness of detail it would be possible to see in this object. But such an inquiry does not even touch the main points towards which an investigation of microscopic vision needs to be directed. It tells us nothing as to what this visual substitute is, how far it can represent something on the microscopic object, and how far it consists of intercostal markings, diffraction-fringes arising from the mismanagement of the illuminating apparatus, or any other misleading effects. And of course it gives us no clue as to how we are either to interpret or control any of these effects.

Every legitimate mode of resolution, and there are numberless such, must lead to precisely the same result, if we can succeed in correctly following out its consequences. Where they differ is in our power of handling them. Abbe's resolution into beams of plane waves recommends itself above all others in regard to this; since it substitutes uniformity for that want of uniformity which exists in all other methods of resolution in just those places where in the present state of our knowledge we are unable to assign the law of this want of uniformity. And even if we ever come to know this law, the resolution into uniform plane waves will still recommend itself in consequence of the law of uniformity being simpler and therefore more easily handled than any law of non-uniformity.

[To be continued.]

XLIII. Galvanometers. By Prof. W. E. Ayrton, F.R.S., and T. MATHER*.

T $N$ an article on "The Electric Discharge in a Magnetic Field" contributed by Sir David Salomons to the Phil. Mag. for September, it is stated on p. 255 :-

"I made a large number of experiments with galvanometers built on the D'Arsonval-Deprez type, and obtained very varying results by modifying the magnetic field. By increasing the field a maximum sensibility was reached, which decreased on further increasing the field.

"The various experiments described no doubt indicate the cause of this, viz. that the field being made too powerful, less current passes through the coil, and the sensibility begins to

* Communicated by the Authors. 